\title{
A Collaborative Video Platform for Learning: From Framework to Action
}

\author{
Peter Tiernan \\ School of Education Studies \\ Dublin City University
}

\begin{abstract}
The work presented in this paper outlines issues relating to the development of a collaborative video platform for learning. Student adoption of collaborative and video technology is increasing dramatically, becoming part of their everyday lives. The aim of this paper is to propose system requirements, a pedagogical framework and design specifications for the successful integration of these technologies into teaching and learning. At the outset we assess current trends and previous research, using these findings to inform the development of a new platform. System specifications are then presented with specific needs identified for students and educators. Our tentative framework for integrating a collaborative video platform for learning is then presented. Finally we establish a course of action for building such a system.
\end{abstract}

\section{Introduction}

The purpose of this paper is to outline our work towards developing a collaborative video platform for learning. Within these pages you will find a justification of our belief that developments in this area are necessary. Included also is a summary of previous research carried out, exploring the impact of findings. Furthermore these results are used to inform the development of our platform, including the pedagogical grounding in using collaborative and video technologies.

We know from previous work (outlined in the following section) that a collaborative video platform for learning has immense potential. However, in order for this potential to be fully realised, the platform must be built on a solid educational framework [1].

The use of video and collaborative technologies has been advancing at pace in recent years, culminating in its use for collaborative learning and creative expression. The challenge that now faces

\author{
Cathal Gurrin \\ CLARITY Centre for Sensor Web \\ Technologies \\ Dublin City University
}

educators is to provide students with a framework that enables them to learn using new media. Meaning they can think, analyse, create, and share information more easily and effectively, using digital media [2]. In meeting this challenge it is imperative that we provide a rich and varied approach to instruction, blending these technologies with traditional teaching strategies, thus providing a well rounded learning environment [3]. We must also recognize that technology alone does not engender innovation. It is instead when technology and instructional pedagogy are fused, that something truly new is created [4]. Over the next number of pages we will establish the background to our work, summarising research which we believe promotes the integration of these tools. We will then introduce our system specifications and learning framework, which are brought to life using collaborative and video technology.

\section{Background}

We began by examining student attitudes to collaborative and video tools currently available. We found that use of social networking sites (SNS) and video sharing sites (VSS) is extremely high, both for personal and academic life. Many students are using these platforms to collaborate on ideas and assignments. Interestingly, students report that while institutional use of technology has a mixed impact on their learning, personal use of SNS and VSS to debate topics and collaborate has a significant one [5]. Kaufman \& Mohan [6] found that while students are becoming more comfortable collaborating with video content online, this has not been met with increased integration into teaching and learning in their institutions. This does not however, as it would initially appear, signify a lack of interest on the part of educators. In fact, educators from across the spectrum are hungry to provide their students with collaborative, ondemand video services. They recognise the merit in facilitating student collaboration and discussion, 
especially around short, focused video clips, suitable for learning. Educators seek more sophisticated ways to integrate these technologies without the current burdens of time, access to content, technical skills and human resources to maintain them [6][7].

We then moved to evaluate video as an instructional tool. Video can increase student motivation and willingness to learn. It encourages interaction with peers and educators, while offering true-to-life scenarios and viewpoints to which students can relate. In the right hands video can spark debate by broadening outlooks and offering different perspectives on topics being discussed [8][9]. In addition, empowering students to become content creators using video helps to draw out their creativity in ways that are not possible using conventional assessment methods [10][20][21]. This act of expression through digital media helps to ensure their potential is reached in a curriculum currently dominated by a single representation of understanding [11]. The ideal environment for learning provides students with the tools to collaborate with one another on common tasks, while also offering them the tools to create exemplary content to share [7].

Having established student utilisation of collaborative tools and the benefits of video for learning, it was pertinent to review previous studies carried out on video services. Initial trials were conducted in Dublin City University using a videoon-demand service with some collaborative features. These features included: content overview, interactive controls, and the ability to create custom video descriptions. The study found both staff and students valued the service. Staff regarded the ability to sort and tag video into relevant clusters for student viewing as an important step forward in linking concepts. Students main attraction to the system was the ability to control their learning, allowing them to pause, rewind and review content. This degree of control allowed time to absorb information and take supplementary notes where necessary. During feedback, students requested a more personalised, social system that would enable them to bookmark, annotate, and share opinions and video clips with peers [12]. A similar trial conducted in Iowa State University found that while making use of the video-on-demand service mandatory, and in particular linking its use to continuous assessment, yielded the greatest results. Students had a similar appetite for more interactive ways of engaging with peers and content [13]. Access to content is also a critical factor, with the best results being achieved when material is available through a web interface both on and off campus. Content should be openly available before and after topics are covered during lectures, giving students time to digest and assimilate information [14].

Video search technology has enjoyed much development in recent years. It is essential to understand how these developments will affect the design and functionally of a new platform. It is now possible to search the entire, media rich content of a video and return a list of relevant, concise video segments for the user to choose from [15]. Using advanced video search techniques (spoken \& written word, annotation, images) reduces the amount of time required to find content, while offering more accurate and targeted results. Content is equally important and a platform must contain the right quantity of news, current affairs and documentary programming in order to appeal to a wide range of users and uses [16]. Recent advances in search design put the power of search into the hands of the user. Techniques such as facial detection and recognition, video segmentation and speech-to-text based searches have huge educational potential linking content by words, imagery and topic [17].

The above research has established that both students and educators value the impact video and collaborative technologies can have on teaching and learning. To realise the untapped potential, strategies must be employed to encourage engagement and interaction with content, educators and peers [18]. Improvements must be made in access to content, organisation of content for retrieval, and assimilation with discussion and collaboration tools. These tools must be brought together using an intuitive user interface that focuses on learning not mastering the technology [19].

\section{System design}

A detailed investigation of the aforementioned literature allows us to bring together the requirements of potential users and develop the following lists of criteria for our collaborative video platform.

\subsection{Search Criteria}

From analysing prior research, we found that barriers to the introduction of video content into teaching and learning include; lack of time to find content, lack of access to content, and lack of resources to manage content. As a result, there are a number of search parameters our platform must offer to alleviate these obstacles. For example, 
users must be able to quickly search by 'content type' to find relevant news, documentary and skills based materials. These searches should also be refinable using 'content descriptors' such as; introduction, summary etc. A full list of search criteria is displayed in Table 1.

Table 1. Search criteria

\begin{tabular}{c}
\hline Content Type \\
\hline Skills demonstrations \\
TV content \\
Experiential viewing e.g. experiment \\
News programming \\
Film \\
Documentary \\
Literature adaptation \\
Research \\
\hline Content Descriptor \\
\hline Introduction \\
Summary \\
Explanation \\
Rated by educator \\
Rated by student \\
\hline
\end{tabular}

\subsection{Educator Criteria}

Prior research also showed that one of the challenges facing educators is to improve students' ability to think, analyse, create and share information using new media, while providing a rich and varied approach to instruction. This approach should blend innovative technologies with traditional teaching strategies to provide a well rounded learning environment. In order to achieve this, educators require 'search criteria' with a sophisticated level of control, for example; filtering content by difficulty and region. They also require input into the 'teaching and learning information', for example; incorporating lesson plans and discussion points. Finally, educators require unique 'system functionality', for example; the incorporation of an "educators area", allowing educators to create and share resources with others. A full list of educator criteria can be found in Table 2 .

Table 2. Educator criteria

\section{Search Criteria}

Short, concise video segments

Ability to tag videos

Search for language learning

Search for local or region specific content

Date range to ensure currency

Search by difficulty level

Search student created work

\begin{tabular}{|c|}
\hline $\begin{array}{l}\text { Discussion points listed within video } \\
\text { content }\end{array}$ \\
\hline Group activities listed \\
\hline Follow-up activities listed \\
\hline Pre-view questions listed \\
\hline Lesson plans provided \\
\hline Clear usage rights and permissions \\
\hline System Functionality \\
\hline Video creation tools \\
\hline Student upload area \\
\hline Share activities with other educators \\
\hline Ability to download and stream videos \\
\hline Ability to create and edit content \\
\hline Create personal collection (favourites) \\
\hline Educator upload area \\
\hline Access off campus/school \\
\hline $\begin{array}{l}\text { Notification when videos of interest are } \\
\text { added }\end{array}$ \\
\hline $\begin{array}{l}\text { Networking opportunities to share } \\
\text { experience and resources }\end{array}$ \\
\hline
\end{tabular}

\subsection{Student Criteria}

In order to fully facilitate student learning, there a number of key criteria the system must meet for the end user. The 'ownership and approach' of the platform is crucial for students. They require a blended learning strategy that allows access to information in a flexible manner, while also allowing the contribution of student content. Our research highlights the importance of 'collaboration features' and as such, the platform should allow students to tag, annotate and highlight video segments for discussion. Also mentioned was the value of advanced user 'functionality', for example; in empowering students to become creators of video content, the platform should support video creation and editing features. A full list of student criteria can be found in Table 3 .

Table 3. Student criteria

\section{Ownership and Approach}

Blended learning approach

Upload user created content

Upload video they have located

Create personal collection (favourites) Web interface for access anywhere 


\begin{tabular}{l}
\hline Functionality \\
\hline Videos available to download \\
Search criteria identical to educators \\
Notification when videos of interest are \\
added \\
High level of control of videos e.g. \\
rewind, pause etc \\
Content overview of videos \\
Intuitive user interface \\
Integrated with LMS to access other \\
documents simultaneously \\
\hline Collaborative Features \\
\hline Tag video \\
Comment on video \\
Share segments of video with others \\
Annotate video \\
Highlight section of video to ask question \\
on it \\
Rank or rate content \\
\hline
\end{tabular}

\section{Core System Functionalities}

While the collation of search, educator and student criteria provides us with a comprehensive view of system requirements, from a systems design perspective it is essential to create a blueprint of core functionalities to be incorporated into our collaborative video platform for learning.

By listing the system requirements outlined above and analysing for common themes, five (5) core functionalities were identified. 1) Search 2) Teaching and learning 3) Web 2.0 4) User and 5) Crawling or gathering. Within these cores we can identify specific functions (fx) for development.

\subsection{Core Search Functionalities}

Advanced search functions are a key success factor for our platform, in order to enhance the learning experience for the end user, the following functions (fx) must be developed: Search (f1), Filter (f2) and summarise/segment (f3)

The system search function (f1) should locate and separate out TV, film, news and documentary programming, so that users can distinguish between factual and fictional programming. The search function (f1) should also include the previously mentioned advanced techniques, such as: spoken $\&$ written word, annotation, images, speech-to-text and facial detection, to locate content. Filtering functions (f2) should enhance accuracy, relevance and suitability for the user by sorting content by date, creator, difficulty and geographical location, with further refinements enabled such as user rating and sequence for learning e.g. introduction to topic or topic summary. Finally and perhaps most importantly, through the use of key frames, the platform should segment and summarise (f3) video content into short concise pieces for the user.

\subsection{Core Teaching and Learning Functionalities}

Teaching and learning functionalities centre on the educators ability to successfully use the system to support learning. Our aim is to embed processes within the system that allow this to happen. This involves four core Teaching \& Learning functions: lesson planning (f4), embedded questions (f5), group activities (f6) and educator sharing (f7).

The first function is the ability to incorporate lesson planning (f4). Educators need to be able to attach and display editable lesson plans so that video lessons and activities can be properly structured and evolve over time. Importantly, this information should only be viewable to educators of sufficient access rights. A second function is the ability to embed pre and post questions (f5) to focus users' attention while viewing video content and promote a sense of inquiry. Third is the ability to implement group activities (f6), by separating users into groups and displaying specific activities for completion. Finally, the system should facilitate the sharing (f7) of information between educators so that sample/suggested questions and activities can be packaged with video content.

\subsection{Core Web 2.0 Functionalities}

The successful integration of core web 2.0 functions in our platform is crucial to bringing about the 'collaborative' aspects and ensuring the learning process is not a series of 'isolated' activities. These functions are: content engagement (f8), user interaction (f9) and content rating (f10).

Content engagement (f8) should encourage users to be actively involved with the learning process. Users should be able to tag, annotate and comment on videos, while also being able to highlight areas of interest and importance and take notes as videos play. Secondly, user interactive functions (f9) should allow users to work together on the video content, sharing interesting or relevant segments with their classmates or highlighting sections of video that are unclear and submitting them to their educator along with questions. Users should also be able to share their notes, annotations and comments with other users. Finally, the ability to rate (f10) content should allow users to express their opinion on the relevance and quality of video 
content, which should in turn lead to better search functions based on user rating. This function should also give users the ability to favourite videos and create a list of favourites.

\subsection{Core User Functionality}

There are a number of functions that are crucial to the overall user experience, these are: user control (f11), creation tools (f12) and notifications (f13).

Users should have a high level of control (f11) over video content in order to take advantage of the power of the video and web 2.0 functions outlined earlier. The ability to pause, stop, rewind, fast forward and bookmark content for later viewing is essential. Bookmarking should be based on user log on so that they can return to where they left of, even if they are using a different machine. The platform should also allow users to download content in addition to streaming. Creation tools (f12) are critical to facilitate users in becoming content creators as well as consumers of video content. Tools should be in place to allow users create, edit and upload video, also allowing them to tag it as relevant to topics. Finally, notifications (f13) should be linked to each users registered account, notifying them of selected changes e.g. videos of interest are added, educator has added an activity or information is received from another group member.

\subsection{Core Gather Functionality}

In order to successfully gather relevant video content and continue to expand the library, the system should be able to: crawl for content (f14) and receive uploads (f15).

Crawling (f14) for content requires the system to store content from terrestrial television stations and sort this into TV, film, news and documentary material. Allowing uploads (f15) will enable the system to gather video content separately from educators and users to build the library, also providing space for user generated content to be shared.

\section{UISCE Framework}

In addition to the technological requirements outlined above, information was used to develop a pedagogical framework to support the integration of our platform into teaching and learning. We believe that a blended learning approach is most effective and that our collaborative video platform can be used in conjunction with, and to augment traditional teaching strategies. Our pedagogical framework, UISCE, highlights what we believe are the most important factors in its integration.

U - Understanding - The overall aim of this framework is to improve teaching and learning. We are not concerned with the promotion of technology, but promoting the development of pedagogical approaches to the use of a collaborative video platform. These approaches are designed to benefit the widest range of students and are outlined below.

I - Inquiry - To take full advantage of the advanced search and collaborative features provided, students should be assigned problems and tasks to complete individually and in groups. These tasks should be exploratory in nature; encouraging students to source and evaluate information, then discuss, debate, and find solutions.

S - Support - Support must be provided through traditional face-to-face teaching approaches. This gives context to collaborative work and provides space for traditional engagement. Support must also be provided during learning using the platform. Tools such as (virtual) notes pads, chat functionality, mind maps and bookmarks, give students the tools they need to learn and collaborate online.

C - Collaboration - Using the tools outlined above students should be given opportunities to work together to complete tasks. Course and assignment work should incorporate working together using the platform to achieve a common goal. Additional collaborative opportunities should be provided by ranking and evaluating video content.

E - Expression - Students should be given the opportunity to express their learning through video and collaboration. Assessments should involve the creation of video artefacts. This allows students to express themselves in new ways and display their creativity. Real learning occurs when students combine lectures, readings, video and collaborative activities into their own video representations.

These factors merge to form our framework, UISCE, which is derived from the Irish word meaning water. It is designed to create a learning environment that promotes the growth of knowledge and understanding, nourishes learning through support and collaboration, and gives rise to new kinds of knowledge through student created content.

\section{From Framework to Action}

Now that the technical specifications and functionalities of our collaborative platform, and 
proposed our underlying pedagogical framework have been outlined, it is important to demonstrate how our theoretical and practical designs align to ensure the best possible teaching and learning experience for users.

Table 4 outlines each of the systems core functions and their impact on our guiding pedagogical framework. We believe this demonstrates our commitment to true educational innovation, where technology and pedagogy are fused.

Table 4. From framework to action
\begin{tabular}{|l|c|c|c|c|c|}
\hline & U & I & S & C & E \\
\hline F1 - Search & Y & Y & Y & & \\
\hline F2 - Filter & Y & Y & Y & & \\
\hline $\begin{array}{l}\text { F3 } \\
\text { Summarise/Segment }\end{array}$ & Y & Y & & \\
\hline F4 - Lesson planning & Y & Y & Y & Y & Y \\
\hline $\begin{array}{l}\text { F5 Embedded } \\
\text { questions }\end{array}$ & Y & Y & Y & Y & Y \\
\hline F6 - Group activities & Y & Y & Y & Y & Y \\
\hline F7 - Educator sharing & Y & & & Y & Y \\
\hline $\begin{array}{l}\text { F8 }- \\
\text { engagement }\end{array}$ & Y & Y & Y & & Y \\
\hline F9 - User interaction & Y & Y & Y & Y & Y \\
\hline F10 - Content rating & & & Y & Y & Y \\
\hline F11 - User control & & & Y & & \\
\hline F12 - Creation tools & Y & Y & & Y & Y \\
\hline F13 - Notifications & & Y & Y & & \\
\hline F14 - Crawl & Y & & Y & & \\
\hline F15 - Receive uploads & & & Y & Y & Y \\
\hline
\end{tabular}

\section{Conclusion}

Over the preceding pages we have demonstrated the need for a framework for the integration of a collaborative video platform for learning. We have shown that student use of these technologies is increasing and that both they and their educators are hungry to use these tools. Through our examination of case studies we have highlighted the benefits of using video in education, drawn out learning from similar trials and outlined future possibilities in the area. We have also proposed a system specification based on student and educator requirements. Finally we introduced our framework for collaborative video learning, identifying the key elements we feel are necessary for both educators and students to fully engage with the system and ensure a positive impact on teaching and learning.

\section{Future Work}

The next step in the process is to construct the platform based on the above system specifications and functions. Trials will then be conducted with a wide range of students to evaluate their experiences with the platform from a useability perspective, its impact on their learning, and their views on learning with digital video and web 2.0 technologies. This will lead to a greater understanding of our platforms ability to promote pedagogically sound teaching and learning.

\section{References}

[1] S. Crump, "e-education: electronic, emotionless and efficient", journal of education policy 14:6, Routledge, 1999, pp. 631-637.

[2] M. Prensky, "H. sapiens digital: From digital immigrants and digital natives to digital wisdom", Innovate 5 (3), Fischler School of Education and Human Services, Nova Southeastern University, 2009.

[3] M.J.M. Farreira, "Intelligent classrooms and smart software: Teaching and learning in today's university", Springer Science and Business Media, Springer publications, 2010.

[4] M. Pearson and S. Naylor, "Changing contexts: Teacher professional development and ICT pedagogy", Springer Science and Business Media, Springer publications, 2006.

[5] S.D. Smith, "The ECAR Study of Undergraduate Students and Information Technology, 2010", Educase, Colorado, 2010.

[6] P.B. Kaufman and J. Mohan, "Video Use and Higher Education: Options for the Future", New York University, New York, 2009.

[7] M.A. Mardis, "Viewing Michigan's digital future: results of a survey of educators' use of digital video in the USA", Learning, Media and Technology 34:3, Routledge, Florida, 2009, pp. 243 - 257.

[8] D. Denning, "Video in Theory and Practice: Issues for Classroom Use and Teacher Video Evaluation", InNATURE Productions, Victoria, 1992.

[9] J.J. Gallagher, "Developing a Heritage and ICT project in 12 Irish Primary Schools, Education and Information Technologies 10:3, Springer publications, Sligo, 2005, pp. 165-175.

[10] M. Papastergiou, "Physical education and sport science undergraduate students as multimedia and web developers: Moving from the user's to the creator's 
perspective", Education and Information Technologies 16:3, Springer, 2011, pp. 281-299.

[11] J.R. Beilke and M.J. Stuve, "A teacher's use of digital video with urban middle school students: Expanding the definitions of representational literacy", The Teacher Educator 39:3, Routledge, London, 2010, pp. 157-169.

[12] C. Gurrin, P. MacNeela, A.F. Smeaton, H. Lee, P. Browne, and K. McDonald, "FISCHLÁR-NURSING, using digital video libraries to teach nursing students", ACM Symposium on Applied Computing, Nicosia, Cyprus, 2004, pp. 1084-1090.

[13] P. Mustillo, P. Bélanger, G. Fayad, and I. Menzies, "A User Interface Design for a Video-on-demand Service Trial in and Educational Setting", European Journal of Engineering Education 22:2, Taylor \& Francis, London, 1997, pp. 135-142.

[14] I.N. Toppin, "Video lecture capture (VLC) system: A comparison of student versus faculty perceptions", Education and Information Technology 16:4, Kluwer Academic Publishers, Massachusetts, 2011, pp. 383-393.

[15] C. Gurrin, "content-based video retrieval", Encyclopaedia of database systems, Springer, USA, 2009, pp. 466-473.

[16] Smeaton, A. Content-based access to digital video: the Físchlár system and the TREC Video track. Paper presented at MMCBIR 2001 - Multimedia Content based Indexing and Retrieval (INRIA, Rocquencourt, France, September 2001.

http://www.cdvp.dcu.ie/Papers/MMCBIR2001.pdf. Last accessed November 2010

[17] P. Ferguson, C. Gurrin, H. Lee, S. Sav, A. Smeaton, N. O'Connor, Y.H. Choi, and H. Park, "Enhancing the functionality of interactive TV with content based multimedia analysis, Multimedia, 2009, pp. 495-500.

[18] N. Davis and D.S. Niederhauser, "Socio-Cultural analysis of two cases of distance learning in secondary education", Education and Information Technologies 10:3, Iowa State University, 2005, pp. 249-262.

[19] R.M. Aiken and J.N. Aditya, "The golden rule and the ten commandments of teleteaching: harnessing the power of technology in education", Education and Information Technologies (2), Springer publications, 1997, pp. 5-15.

[20] Y. Crotty, "Through the enlightened eye and I am I brining creativity and visual literacy into Higher Level Education?", Educational Journal of Living Theories 4:1, 2011. Available from http://ejolts.net/node/184

[21] Y. Crotty and M. Farren, "Promoting creativity in higher education", Encouraging creativity in education: a handbook for current and future teachers, 2009, pp. 120 125 . 